\title{
FÚTBOL FEMENINO: ¿RUPTURAS O RESISTENCIAS?
}

WOMEN'S SOCCER: BREAKING OR RESISTANCE?

Jorge Humberto Ruiz Patiño ${ }^{1}$

\section{Resumen}

Con la escogencia del fútbol femenino como objeto de investigación se pretende indagar sobre las relaciones entre feminidad y fútbol, teniendo en cuenta que la participación femenina en el fútbol plantea cambios referentes a las formas generales de representación de la feminidad, específicamente en lo tocante a las formas hegemónicas de portar el cuerpo. A partir del análisis concreto de los cambios que se observan en el ámbito de la propia práctica futbolística, se observan transformaciones que sobrepasan el interés específico deportivo y que indican modificaciones en algunas disposiciones de apreciación y percepción tanto femeninas como masculinas. Al mismo tiempo, permite comprender las resistencias estructurales que se presentan a las transformaciones producidas por esta práctica y que sirven también como indicadores de dinámicas más amplias de la sociedad colombiana. Las siguientes páginas proponen una interpretación del fútbol femenino que, partiendo de una concepción general de la modernidad y del sujeto social, aborda el fenómeno desde la práctica local del fútbol, específicamente en la Universidad Nacional de Colombia, tomando como elementos centrales el cuerpo, el juego y la competencia. Estos elementos son articulados mediante la categoría de género y la teoría de Pierre Bourdieu, específicamente los conceptos de campo, capital y habitus.

Palabras clave: fútbol femenino, género, cuerpo.

\section{Abstract}

By choosing women's football as a research object we intend to explore the relationship between femininity and football, considering that female participation in football poses changes related to general forms of representation of femininity, regarding specifically the hegemonic ways to carry the body. From a concrete approach to the changes observed in the very football practice, we evidence changes that go beyond the sports specific interest and indicate changes in some appreciation and perception dispositions regarding both female and male. At the same time, provides insight into the structural resistance to the changes occurred in this practice and which serve also as indicators of broader dynamics of Colombian society. The following pages offer an interpretation of women's football, from a general conception of modernity and the social subject, analyzing the phenomenon from the local soccer practice, specifically in the National University of Colombia, taking body, play and competition as central elements, articulated here through the category of gender and the theory of Pierre Bourdieu, particularly the concepts of field, capital and habitus..

Keywords: women's soccer, gender, body.

Fecha de recepción: 6 de Julio de 2011

Fecha de aprobación: 18 de octubre de 2011

\footnotetext{
1 Magíster en Estudios Políticos de la Pontificia Universidad Javeriana, Sociólogo de la Universidad Nacional de Colombia, docente de la Universidad Pedagógica Nacional, miembro de la Asociación Colombiana de Investigación y Estudios Sociales del deporte -ASCIENDE-. Correo electrónico: jorge5ruiz@yahoo.com.mx
} 


\section{Introducción: fútbol, espacio y hegemonía}

La hegemonía del fútbol en el campo deportivo resulta ser una alegoría del proceso hegemónico de la razón instrumental en occidente. En efecto, aunque el fútbol es hijo de la modernidad, como todos los demás deportes, ninguno representa mejor a la modernidad triunfante como aquel. Este deporte que surge, según Norbert Elías (1996), como producto de los procesos de pacificación interna del Estado en la sociedad inglesa, se erige como un espacio fundamental de la manifestación pública de las élites, es decir, se convierte en el medio por el cual el hombre burgués, representante típico de la modernidad y del capitalismo occidental, manifiesta su carácter moral en el tiempo libre.

El fútbol, como cualquier otra práctica social, hace parte de la división moderna del espacio social en las esferas pública y privada, con la subsiguiente dicotomía homóloga de masculino/femenino (Amorós, 1994). Pero asimismo el espacio está divido en tiempo libre y tiempo ocupacional, por llamarlo de alguna manera. En este último, las actividades nuevamente se encuentran divididas según las dicotomías de público/privado y masculino/femenino y existe una correspondencia de las actividades públicas con las masculinas y de las privadas con las femeninas. De la misma manera, las actividades del tiempo libre comparten esta taxonomía social; los deportes se ubican en el espacio público y son desempeñados específicamente por los hombres, mientras que las actividades de tiempo libre de las mujeres se desarrollan en espacios privados.

Lo específico en los deportes que hace que estos sean una práctica pública de tiempo libre es la exposición corporal como instrumento de distinción y manifestación del carácter moral. En este sentido, el cuerpo en los deportes femeninos ha sido significado de manera tal que corresponde con las cualidades morales asignadas culturalmente a las mujeres, es decir, cualidades como la sensibilidad, la fragilidad, etc. Esto quiere decir que la exposición corporal de la mujer a través de los deportes se encuentra significada de forma diferente a la exposición corporal masculina. Sin embargo, lo que hace que lo público corporal sea detalladamente masculino es la estructura de poder entre los sexos y entre los mismos deportes, los cuales constituyen campos diferenciados. Todo esto significa que aunque las mujeres realizan una exposición pública en los deportes practicados por ellas, el significado de su práctica, o la representación social que se hace de ella, se minimiza respecto a su importancia como práctica pública en la cual se definen situaciones relevantes de la sociedad.

La dicotomía público/privado hace parte de todo el sistema de parejas dicotómicas que proporcionan un marco simbólico de interpretación a las diferencias biológicas entre los sexos y que determinan las categorías de juicio sobre las cualidades, aptitudes y actitudes características de cada sexo (Bourdieu, 2000). Así, lo público se asocia con todas las cualidades físicas y morales del hombre, y lo privado con todas las cualidades físicas y morales de la mujer. En este sentido, el deporte, y el fútbol particularmente, podrían asociarse con la esfera pública en la medida en que la dinámica interna de éste, el desarrollo del juego, concuerde con los estereotipos fijados para el sexo masculino. Además, el fútbol pertenece a una esfera de la sociedad en donde se definen apuestas importantes de los grupos, tales como el honor y las distintas representaciones que se pueden derivar de la victoria de un grupo o individuo sobre otro, pues desde la cultura griega la victoria sobre un contrincante ha significado un aumento en el prestigio y por ende en las posibilidades de mejorar la posición en la escala social jerarquizada (Elias, 1996). Así mismo, los juegos de carácter físico han tenido la característica, en muchas sociedades, de ser espectáculos (no en el sentido capitalista) observados por una cantidad específica de individuos que con intereses diferentes, según la época y la sociedad, esperan el resultado del juego como un imperativo del mismo; en este sentido, el carácter público del deporte es indiscutible. En el fútbol, por su lado, se pueden definir apuestas como el honor, el mercado económico, la supremacía étnica, política y social, etc, como derivadas de una constante exposición del cuerpo a través del dominio de una técnica específica.

El fútbol se ha configurado como el paradigma deportivo de la modernidad. Su hegemonía como práctica deportiva está dada por la influencia de varios factores. De un lado la estructura de poder entre los sexos permite que un deporte masculino, como el fútbol, se imponga sobre los demás deportes de tipo femenino. En este sentido, es claro que si el cuerpo masculino está dotado de cualidades públicas que le permiten un mayor desempeño en esta esfera, los deportes identificados con lo masculino estarán en la cima de la jerarquía entre todos los deportes, así como será mayor su importancia en la definición de asuntos relevantes para la sociedad. Otro aspecto importante en la definición del fútbol como deporte hegemónico es su marcada difusión frente a otros deportes de tipo masculino, que 
logra su máxima expresión con el auge de los medios masivos de comunicación, específicamente a partir de las transmisiones televisivas de los mundiales, cuyo ejemplo más elocuente es el campeonato mundial realizado en México en 1970.

Así las cosas, la estructura de poder entre los sexos, la conformación de un espacio público deportivo (y masculino) y la transmisión global de los campeonatos de fútbol han permitido que el fútbol se erija como el deporte hegemónico. Podría decirse que el fútbol es al campo deportivo lo que el Estado es a la sociedad, es decir, que el Estado y el fútbol son los hijos predilectos de la modernidad occidental. Ningún otro deporte como el fútbol reclama para sí carácter de universalidad derivado de su cualidad global así como de la definición masculina que posee. De otro lado, al igual que el Estado, el fútbol es el espacio predilecto de las disputas públicas, ya que es el deporte con mayor visibilidad, difusión y el que permite, desde el punto de vista simbólico, mayores ganancias políticas. En el espacio público del tiempo libre el fútbol ocupa un lugar de predominancia frente a los demás deportes ya que ciertas apuestas de la sociedad se definen desde el fútbol como espacio de mayor significado público.

Ante estos planteamientos surgen las siguientes preguntas: ¿Cuál es el sentido de las transformaciones sucedidas cuando el espacio público del fútbol es ocupado/disputado por las mujeres? ¿Qué sucede con el estatus de la dicotomía público/privado y con las representaciones de lo femenino una vez se fractura la rigidez de dicha dicotomía? Los apartes siguientes tratan de contestar estas preguntas desde dos perspectivas conexas. La primera busca aclarar el problema a partir de una reflexión que activa la pregunta por las posibilidades de existencia del fútbol femenino a nivel macro, relacionando las nociones de globalización y sujeto como elementos centrales de la emergencia pública de las mujeres en los deportes, todo esto en el contexto de múltiples crisis asociadas a la modernidad triunfante. Esta reflexión sirve de entrada a la segunda perspectiva, que busca aclarar los problemas antes formulados partiendo del contexto micro y tomando como base el estudio de caso Representaciones de género en el fútbol femenino: estudio del fútbol femenino en la Universidad Nacional de Colombia realizado durante el año 2003. Esta investigación, si bien puede presentar contrastes derivados del balance histórico de los últimos años, en los cuales la práctica del fútbol femenino ha cobrado mayor relevancia y difusión, tiene validez actual en el sentido que proporciona un modelo de análisis para las investigaciones contemporáneas que traten de ubicar las disyuntivas en las construcciones de feminidad en torno a la práctica del fútbol. El estudio de caso presenta un balance de estas construcciones en torno al surgimiento de un nuevo sujeto local que pone en escena una experiencia novedosa del cuerpo, la cual es atravesada por procesos de resignificación, resistencia y negociación de las imágenes hegemónicas y emergentes del cuerpo femenino en la esfera pública del fútbol.

\section{Globalización, fútbol y crisis: el preludio del suje- to femenino en el fútbol}

Como consecuencia de la crisis de la modernidad hegemónica, la razón instrumental y el Estado, el sujeto femenino en el fútbol ${ }^{2}$ surge -tardíamente- en la década de 1990. La fractura en los estereotipos de género asociados a la modernidad hegemónica permite la creación de significados alternativos del cuerpo que construyen la posibilidad de la práctica futbolística femenina, lo que configura una crisis en el fútbol como deporte representativo de la masculinidad. Esto quiere decir que si la emergencia del sujeto social contemporáneo hace manifiesta la crisis de la modernidad hegemónica y del Estado, el surgimiento del sujeto femenino en el fútbol representa la crisis de esta práctica como deporte hegemónico. Si el Estado y el fútbol son hijos predilectos de la modernidad, su crisis se manifestará de manera palpable en los dos, aunque en tiempos diferentes.

La globalización ${ }^{3}$ actúa como el contexto general de la crisis del fútbol y de la posibilidad del sujeto femenino, cuya fuerza motora es la fractura de la modernidad. La conectividad compleja de la cultura en el marco de la globalización (Tomlinson, 2001) introduce la idea de reflexividad, que permite explicar la formación del sujeto femenino del fútbol en el contexto global. Retomando a

2 Esto no quiere decir que con anterioridad no hayan existido formas de fútbol practicado por mujeres, como es el caso del British Ladies Football Club fundado por Netie Honeyball en 1894 y el Dick, Kerr Ladies F.C. fundado en la ciudad de Preston durante la primera guerra mundial. De lo que se trata aquí es del sentido sociológico de la posibilidad de un proceso de subjetivación consistente y permanente, además de transformador de estructuras profundas.

3 Entendemos globalización como "la intensificación de las relaciones sociales mundiales que enlazan sitios distantes de forma tal que los sucesos locales están influidos por acontecimientos que ocurren a muchos kilómetros de distancia y viceversa". Giddens, citado por Tomlinson (2001, p. 54) 
Giddens, Tomlinson (2001) plantea que la reflexividad es la cualidad que los agentes modernos tienen para examinarse y reformularse constantemente mediante una relación dialéctica entre los agentes individuales e institucionales:

Es esta sensibilidad reflexiva de las instituciones ante la información de los agentes humanos lo que caracteriza el dinamismo peculiar de la vida social moderna y define la conectividad entre una multiplicidad de actos locales individuales mínimos y las estructuras y los procesos globales más generalizados (p. 29).

La noción de reflexividad impide que la conectividad cultural de la globalización sea un proceso unilateral; por el contrario, piensa la globalización como un proceso doble, dialéctico, donde actúan agentes locales y globales, significándose los unos a los otros, reelaborando cada una de sus dinámicas. De esta forma, así como las acciones globales repercuten en lo local, las acciones locales tienen la capacidad de provocar consecuencias en el ámbito global. Para el caso del sujeto femenino en el fútbol, el movimiento entre lo local y lo global se puede definir más o menos de la siguiente manera: las presiones comienzan a nivel local con la decisión de algunos grupos de mujeres vanguardistas europeas de jugar fútbol y de organizar torneos. Este ejercicio local presiona una demanda en las altas esferas del fútbol asociado, con lo que se logra la incursión del fútbol femenino como práctica y deporte relativamente reconocido dentro del campo del fútbol. Aquí se sobrepasa el nivel local y se logra un desarrollo global del fútbol femenino con la organización de torneos mundiales y continentales especialmente en Europa. Lo global realiza una labor de difusión de este nuevo deporte, mediante la acción de los medios masivos de comunicación, y crea en lo local la posibilidad cultural, a través de la conectividad, del fútbol femenino en zonas donde esta modalidad deportiva, por diversas condiciones culturales, económicas o sociales, no había tenido un desarrollo comparable con el europeo. Así, se puede observar un movimiento continuo que pasa de lo local a lo global y viceversa, en un proceso reflexivo que no se detiene.

Siguiendo a Bourdieu (1988), y retomando la noción de reflexividad, podemos decir que los cambios en las prácticas deportivas de las mujeres se deben a unos cambios en los gustos, es decir, a un cambio correlativo entre los campos de consumo y de producción (Bourdieu, 2000a). La presión ejercida por las mujeres en el contexto de los cambios generales en su condición, es decir, las nuevas concepciones acerca de ser mujer y de sus posibilidades en la sociedad, debió haber incidido en el campo de producción deportivo, pues la demanda, de alguna manera, exige las condiciones de su realización creando la posibilidad lógica y real (concreta) de ofrecer unos bienes y unas prácticas que con seguridad van a ser apropiados por los agentes en cuestión. En efecto, los agentes del campo de producción no tendrían en sus planes producir unos bienes deportivos si no tuvieran la seguridad de que estos fueran a ser consumidos. De otro lado, en el campo del consumo, el gusto, definido como "propensión y aptitud para la apropiación de una clase determinada de objetos o de prácticas" (Bourdieu, 1988: 172), encuentra su homólogo en los nuevos bienes futbolísticos ofrecidos a las mujeres, gracias a las nuevas disposiciones que permiten que las mujeres propendan por desarrollar una práctica que otrora no era apta para ellas.

La formación del campo del fútbol femenino conlleva transformaciones fundamentales en la estructura social entre los sexos. Estas transformaciones hacen parte de cambios más generales en las relaciones de género donde los discursos hegemónicos de la masculinidad y la feminidad se han fragmentado, lo que permitió el surgimiento de nuevas formas de vivir y portar el cuerpo y la sexualidad. La fragmentación (relativa) en las formas monolíticas de poder en las relaciones entre los sexos hace parte del proceso que, en términos de Alain Touraine (1992), recupera al sujeto perdido de la modernidad triunfante. Para Touraine, el sujeto y la racionalidad nacen como parte del mismo proceso de la modernidad. Sin embargo, la técnica, la racionalidad y la ciencia se convirtieron en los elementos que definieron la modernidad occidental y ocultaron grupos sociales cuyas formas de acción alternaban con las formas hegemonizadas del individuo racional y capitalista de la modernidad.

La crisis de la razón, representada de manera más concreta en el totalitarismo de Estado, produce el resurgimiento del sujeto como una forma de resistencia a la hegemonía de la razón instrumental, para la cual las formas objetivas de acción son universales y por tanto verdaderas. El sujeto recupera al individuo para convertirlo en actor de su propia transformación más allá de definiciones trascendentales impuestas sobre las sociedades y sus grupos, pues según Touraine

La subjetivación es lo contrario del sometimiento del individuo a valores trascendentes: antes, el hombre se proyectaba a Dios; en adelante, en el mundo moderno, es el hombre quien se convierte en fundamento de los 
valores, puesto que el principio central de la moral es la libertad, una creatividad que es su propio fin y se opone a todas las formas de dependencia (Touraine, 1992: 209).

Entonces, las recientes transformaciones en las relaciones de género hacen parte de todo este proceso de crisis de la razón instrumental y del resurgimiento del sujeto. Los movimientos de mujeres, desde diferentes perspectivas, han definido nuevos roles diferentes de los papeles asignados tradicionalmente a las mujeres en el marco de una ideología de lo masculino. Con la fractura de la modernidad se fracturan también los roles hegemónicos de género, por lo cual surgen sujetos femeninos y masculinos que reelaboran cosmovisiones del cuerpo y de la vida.

El fútbol femenino surge como parte de la crisis de hegemonía, pero también de la formación del sujeto femenino, ya que no se puede explicar la ruptura que plantea un uso diferente del cuerpo sin tener en cuenta la crisis de la razón y de los roles sociales creados y definidos por ella. El sujeto del fútbol femenino emerge de la profunda crisis de los imaginarios de género definidos y consolidados durante el proceso de triunfo de la modernidad racionalizadora. Sin embargo, la aparición de este sujeto sucede de manera retardada teniendo en cuenta la época de surgimiento de los primeros movimientos feministas, los cuales buscaron cambios prioritarios en las estructuras de la acción social y política. A pesar de las fuertes presiones por modificar los usos culturales del cuerpo, la incursión de las mujeres en deportes definidos desde los principios de la modernidad como masculinos es muy reciente, evidenciando, en términos de Bourdieu (1988), la estructura jerárquica de los campos de acción social.

El fútbol femenino recupera a la mujer como agente activo y transformador de su realidad, es decir, como sujeto. Implica el derrumbamiento de instancias trascendentales impuestas sobre el individuo, el cual se convierte en parte de la estructura pero no en actor de la misma. El sujeto femenino transforma a las mujeres al tiempo que transforma al fútbol, convirtiendo una realidad unívoca con pretensiones universales y trascendentales en una realidad de múltiples y flexibles significaciones; una realidad que no se impone sino que es construida por los diferentes actores.

Así, lo público en el tiempo libre se transforma, procurando espacios de exposición corporal heterogéneos. Lo público deja de ser un atributo directamente relacionado con lo masculino permitiendo al sujeto femenino encontrar alternativas de intercambios simbólicos más equitativos, participando activamente en las apuestas simbólicas relevantes de la sociedad. En síntesis, el sujeto femenino en el fútbol define nuevos horizontes éticos y morales para las mujeres, es decir, mayores capacidades en la búsqueda de definiciones significativas de orden público, que en el espectro del tiempo libre difieren de las definiciones tradicionales dentro de la cuales se enmarcaban las mujeres y que marcaban un espacio público restringido y rezagado de las apuestas imperantes.

\section{El sujeto local: estudio de caso}

En este aparte se presentan los resultados del estudio de caso Representaciones de género en el fútbol femenino: Estudio del fútbol femenino en la Universidad Nacional de Colombia (Ruiz, 2003), como una forma de dar sustento empírico a las reflexiones presentadas anteriormente. La investigación buscaba indagar en un caso concreto y específico de práctica femenina del fútbol, la manera como podían transformarse las representaciones dominantes del cuerpo en relación a la construcción de la idea de feminidad. El problema se abordó desde la teoría de Pierre Bourdieu con los conceptos de campo, habitus y capital, marco teórico que, a partir del análisis del discurso aplicado a una serie de entrevistas realizadas, permitió identificar la estructura del campo del fútbol en la Universidad Nacional y un conjunto de capitales específicos que definieron prácticas y significados diferenciados sobre la práctica femenina del fútbol. A continuación se presentan los resultados de la investigación haciendo omisión de los textos de las entrevistas para una mayor síntesis.

El caso del fútbol en la Universidad Nacional de Colombia se puede delimitar como un microcampo en el que se dan nuevas relaciones con la incursión del fútbol femenino. Con anterioridad a esta incursión, el campo del fútbol en la Universidad Nacional estaba configurado como un espacio de luchas entre hombres por la acumulación de un capital deportivo-futbolístico, conformado por técnicas y victorias que otorgaban legitimidad al fútbol jugado por un equipo o por un jugador en particular. En últimas, las luchas en este microcampo se daban por la definición de quién jugaba mejor, tanto colectiva como individualmente.

La llegada del fútbol femenino trae pareja la aparición de un nuevo tipo de agente (sujeto) que entra a competir por el capital en cuestión. El campo del fútbol en la Universidad Nacional entra en una nueva dinámica 
proporcionada por el nuevo agente femenino que trae consigo nuevas formas de lucha y nuevas especies de capital útiles para lograr la legitimidad de su práctica.

En efecto, las mujeres, por ser un tipo de agente distinto de los hombres en cuanto a sus condiciones de existencia, ponen en juego nuevos significados del fútbol que tienen que ver con sus esquemas de percepción, con lo que dan un nuevo sentido a las luchas por el reconocimiento de la práctica dentro de este microcampo. De esta forma, entran en competencia las definiciones femeninas y masculinas de la práctica futbolística. Dentro de esta lucha se observa la acción de dos especies de capital apropiados diferencialmente por los agentes de este campo: capital deportivo-futbolístico y capital corporal.

Las mujeres entran en el campo como un nuevo agente que debe ganarse el derecho de participar dentro de él. La legitimidad de su práctica está determinada no por la tradición o la trayectoria, sino por la capacidad que tenga de acumular el capital imperante dentro del campo. La lucha de este agente por hacer valer su actividad futbolística como digna de pertenecer al campo depende también de las posibilidades objetivas que existan dentro del éste para apropiarse fructíferamente del capital que define la legitimidad de la práctica. La mujer ocupa, por tanto, una posición subordinada en el campo del fútbol, pues como agente pretendiente dentro de él no posee la competencia tradicional que le da el derecho de hacer valer su actividad sobre la actividad de otros agentes.

Según Bourdieu (1988) la fórmula generadora de la práctica social es la siguiente: [(habitus) (capital)] + campo $=$ práctica. Siguiendo esta relación la práctica del fútbol femenino y las representaciones que se puedan dar en él, dependen tanto del capital imperante como de los habitus de cada agente y la dinámica del campo. De esta forma el fútbol femenino tendrá una significación diferente del masculino y las prácticas futbolísticas de cada agente estarán en lucha por la legitimidad.

Cuando se habla de campo futbolístico, así como de cualquier otro campo, la primera cuestión a plantear se centra sobre la determinación de qué es lo que está en juego dentro de ese campo, cuáles son las apuestas por las que los agentes se enfrentan en una lucha por la legitimidad y cuál es su distribución entre los agentes. El capital deportivo-futbolístico es la configuración específica de apuestas imperantes dentro de este campo. Este capital es la apuesta principal, aunque no determinante, como se verá más adelante, con la que los agentes se enfrentan dentro del campo y orientan sus acciones futbolísticas. Puede ser definido como el conjunto de recursos técnico-tácticos con que cuentan los agentes futbolísticos además del nivel de desempeño que logran los agentes tanto colectiva como individualmente.

A primera vista, partiendo de algunas observaciones generales, el capital futbolístico no está simétricamente distribuido entre los géneros en el campo de la Universidad Nacional. El nivel técnico de las mujeres futbolistas está desfavorablemente ubicado con respecto al nivel observado para los hombres futbolistas. Esto indica una posición subordinada dentro del campo por parte de las mujeres, ya que el capital imperante de este campo no ha sido apropiado de igual manera ni en igual cantidad que los hombres.

Para participar en el campo del fútbol las mujeres han tenido que aplicar un cierto nivel de técnica y de táctica que les permita convertirse en actores activos del campo. Es cierto que se ha apropiado y acumulado una parte del capital específico, pues de todas formas, para pertenecer al campo es necesario aprehender los imperativos de aquel. Sin embargo, ese capital apropiado por las mujeres está determinado por la configuración histórica del campo y la participación de las mujeres en él.

$\mathrm{Al}$ estar en lucha con los agentes masculinos y al no poseer la competencia técnica, las mujeres contraponen otra forma de experimentar el fútbol con la pretensión de que ésta nueva experiencia sea tan valorada como la de los hombres. Así, contraponen una percepción del cuerpo externo, como la técnica, a una valoración del cuerpo interno. La técnica y la táctica conforman una relación con el cuerpo en la que se expresa, mediante diversos movimientos corporales, un dominio del cuerpo que implica que el que posea ese dominio se erija simbólicamente como un sujeto superior, no sólo en cuestión del dominio de la técnica, sino también como una manifestación de superioridad moral (Pedraza, 1999). $\mathrm{Al}$ no poseer la facultad de expresar alguna superioridad a través del dominio del cuerpo en cuanto al fútbol, el cuerpo interno parece ser una buena opción para suplir la falencia técnica.

Hacer valer la propia práctica con un nuevo sentido implica también hacer funcionar una nueva especie de capital dentro del campo. Lo recreativo aparte de ser un discurso de la práctica femenina funciona también como contraparte del imperativo tradicional de lo competitivo en el fútbol. En otras palabras, para entrar en el campo 
del fútbol como agente femenino es necesario jugar la carta de lo recreativo como apuesta principal. No es que las mujeres no apropien el capital imperante (deportivofutbolístico), pues de otra forma no podrían actuar en el campo del fútbol. El agente femenino posee un cierto nivel y un cierto conocimiento de la táctica y cada vez va acumulando mayor cantidad de estos capitales, pues el imperativo de la competencia y la agresividad también es un capital que poco a poco se va apropiando. De otro lado, este nuevo sentido del juego hace que surjan representaciones respecto a un estilo de juego diferente como una concepción del cuerpo externo o una manifestación objetiva del cuerpo. Lo lúdico en el fútbol, lo recreativo, crea la imagen objetiva de una forma de jugar que se diferencia de la forma masculina y que se trata de imponer de igual forma como se trata de imponer el sentido (interno) recreativo del fútbol.

Así, cuando se tiene la percepción de que no hay bastante capital deportivo-futbolístico acumulado se pone como apuesta el sentido recreativo, y paralelamente, cuando si existe la constancia de la apropiación del capital deportivo-futbolístico se contrapone un estilo de juego femenino, más puro, libre de toda imperfección violenta y con mayor claridad en los movimientos técnicos. En definitiva, lo que se contrapone son concepciones diferenciales referentes al cuerpo interno (sentido y significado) y al cuerpo externo (estilo). Además, el estilo de juego femenino aparece como una cualidad artificial, preparada, construida externamente, mientras que en los hombres es algo "instintivo", es una cualidad que aparece naturalizada, innata en el cuerpo masculino.

La relación del fútbol (y cualquier deporte) con el sujeto social, es la relación de éste último con su propio cuerpo como entidad experimentada subjetivamente en la aplicación de una técnica, es decir, en el dominio del cuerpo con un fin específico. Pero también es la experiencia vivida socialmente, experiencia objetiva basada en la producción e intercambio de significados materializados en la corporeidad humana. El cuerpo es una entidad subjetiva, vivida como propia, privada, pero a la vez está marcado por lo colectivo y lo comunicativo, y de esta forma, es cuerpo percibido, observado y comparado. Estas dos dimensiones del cuerpo, lo subjetivo y lo social (Rodó, 1994), están atravesadas por las construcciones culturales de las que aquel es objeto, es decir, por significados adjudicados en el marco de un proceso histórico de socialización de lo biológico (Bourdieu, 2000b). El fútbol, por tanto, es una relación fundamentalmente corporal.

Pero al tiempo que las mujeres adoptan facultades que histórica y simbólicamente han sido apropiadas por el género masculino, realizan una reinterpretación del juego y por ende de su cuerpo a partir de las condiciones de apropiación del capital deportivo-futbolístico y de los esquemas de percepción y apreciación del habitus. Ahora existen dos visiones del fútbol que luchan por ser reconocidas dentro del campo futbolístico:

Debido al hecho de que los agentes aprehenden los objetos a través de los esquemas de percepción y apreciación de sus habitus, sería ingenuo suponer que todos los practicantes de un mismo deporte atribuyen el mismo sentido a la práctica o incluso suponer que practican, propiamente a hablando, la misma práctica (Bourdieu, 1988: 208).

Estas dos visiones, o estas dos prácticas futbolísticas, son el resultado del desarrollo de dos condiciones específicas de existencia que constituyen uno de los factores en la distribución del capital imperante dentro del campo entre los agentes.

Entonces, en el fútbol femenino se da una resignificación del cuerpo a partir de la adopción de nuevas formas de sentir y presentar el cuerpo en el contexto de la contienda deportiva. Esta nueva presentación del cuerpo constituye una forma diferente de exposición a través de un lenguaje tradicionalmente masculino, lo que puede ocasionar en muchas ocasiones efectos sobre las representaciones imperantes de feminidad. La paradoja del fútbol femenino consiste en que la conquista o incursión de un espacio estratégico de la sociedad como es el fútbol conlleva en sí misma una disminución de la imagen legítima que se tiene del cuerpo femenino, tanto subjetiva como objetiva.

Lo que nos puede enseñar el análisis del capital corporal, además de las posibilidades objetivas de apropiación del capital deportivo-futbolístico, son las imágenes o las representaciones del cuerpo femenino que surgen a raíz de la participación activa en el campo del fútbol. La feminidad en el fútbol es un concepto que varía según la configuración de los capitales corporal y deportivofutbolístico. Una relación clara entre estos capitales enseña que el corporal incide sobre el significado de la práctica futbolística, es decir, sobre el capital deportivo-futbolístico, mientras que la posesión de este último incide sobre las visiones del cuerpo, es decir, sobre el capital corporal, y de esta forma, ambos capitales inciden determinantemente sobre la práctica futbolística en su conjunto.

En primer lugar, parece como si la participación en el campo del fútbol, la posesión de un determinado nivel 
de capital deportivo-futbolístico, produjera una desinversión del capital corporal, es decir, una disminución de la valoración de la imagen, tanto objetiva como subjetiva, del cuerpo femenino:

Las oportunidades de vivir el propio cuerpo bajo el modo de la gracia y el milagro continuo son tanto mayores, en efecto, cuanto más a la medida del reconocimiento es la capacidad corporal; o, a la inversa, la posibilidad de sufrir el cuerpo en el malestar, la incomodidad, la timidez, es tanto más fuerte cuanto mayor es la desproporción entre el cuerpo ideal y el cuerpo real (Bourdieu, 1988: 204).

Los efectos de la posesión del capital deportivo-futbolísitco pueden ejercerse negativamente debido al desajuste entre la visión hegemónica del cuerpo y las nuevas formas que el juego del fútbol propone.

De otro lado, los aspectos simbólicos del juego del fútbol en cuanto al dominio de una técnica, otrora potestad exclusiva de los hombres, se notan en la percepción del cuerpo como una entidad trastocada de su esencia. El monopolio de la técnica en manos de los hombres es tan fuerte, que el estereotipo dominante del cuerpo femenino no permite que la mujer adquiera una técnica futbolística sin que su cuerpo sufra una trasformación simbólica de sus formas. Lo que está en el fondo de todo esto es la acción profunda de los esquemas clasificadores de los habitus y de las posibilidades de apropiación del capital futbolístico.

En resumen, el fútbol produce casi de inmediato, y en muchas formas, un efecto de desinversión del capital corporal imperante en el campo de la definición de los usos legítimos del cuerpo. Es de notar que este es un microcampo con particularidades propias donde se define la visión dominante de cuerpo. En términos generales, la visión del cuerpo de los agentes implicados en el campo del fútbol de la Universidad Nacional se opone a un cuerpo femenino grueso o grande, agresivo y fuerte. Se puede observar un cuerpo femenino dominante que se representa delgado, un poco frágil, sin tanta fortaleza, y sin expresiones de agresividad o de esfuerzo físico. La desinversión primaria del capital corporal es directamente proporcional a una mayor posesión de capital deportivo-futbolístico, es decir, a una mayor inclusión y participación en el campo del fútbol. El cuerpo femenino recibe la acción de dos campos de poder; de un lado, es necesario ser eficiente dentro del campo del fútbol, y para lo cual se hace imperativo acumular capital deportivo-futbolístico, mientras que del otro extremo se debe mantener y reproducir un capital corporal dominante, el cual mantiene una relación inversa con el capital deportivo-futbolístico. Aquí el cuerpo femenino parece ser ambivalente y a veces contradictorio; ambivalente porque recibe el valor de dos campos diferentes que se mezclan y en los cuales debe ser lo suficientemente eficiente; y contradictorio por que esos dos campos en los cuales participa activamente poseen capitales simbólicos repelentes, casi con un carácter antitético.

Al advertir los efectos de desinversión en cuanto capital corporal, producidos por el flujo simbólico del campo de fútbol, las mujeres responden con una fuerte re-inversión de este capital en sus aspectos más característicos. La fuerza simbólica ejercida por el campo del fútbol actúa como un incentivo para que las mujeres aumenten o hagan más visibles los aspectos de su cuerpo pertenecientes a una definición legítima de feminidad dentro del contexto en el que actúan. Una re-inversión de capital corporal significa seguir siendo eficientes, siguiendo ciertos límites, dentro del campo del fútbol, a la vez que se mantiene y se reproduce la visión hegemónica del cuerpo femenino; como ya se dijo, el mantenimiento del capital corporal limita las posibilidades femeninas de lograr una mayor eficiencia dentro del campo futbolístico. Así, los aspectos de la feminidad arraigados en el cuerpo se resaltan con mayor ahínco dentro de la cancha de fútbol.

El problema central de este asunto radica en que los capitales antitéticos son tales porque sus poseedores naturales son antitéticos entre sí mismos. El capital deportivo-futbolístico se convierte en capital simbólico al ser re-conocido, es decir, al considerarse legítimo dentro del campo y también al considerarse legítimos sus poseedores. El re-conocimiento de un capital indica una naturalización de su posesión, es decir, una condición innata de las competencias necesarias para actuar en el campo. El capital deportivo-futbolístico es un capital simbólico poseído por el género masculino; su competencia es una facultad dada por la naturaleza masculina, es decir, la técnica futbolística pertenece al conjunto de los comportamientos naturales masculinos.

\section{Conclusión}

El cuerpo femenino en el fútbol posee una doble cualidad y valor. El sujeto femenino se debate entre dos campos de fuerza: el campo del fútbol y el campo de las luchas por la definición del cuerpo legítimo. En el momento en que una mujer entra a una cancha de fútbol su cuerpo se bifurca simbólicamente, cobra una 
doble esencia. Esta dualidad del cuerpo femenino está dada por la imagen formada de la mujer como una actor nuevo y extraño dentro del fútbol; la extrañeza se justifica en que la mujer no es el actor legítimo de este campo o por lo menos todavía no lo es, no posee autoridad y por tanto carece de capital simbólico; es decir, no posee la naturalidad dentro del campo. Por el contrario, la naturalidad masculina deviene de su larga participación y de la adecuación de su cuerpo con las exigencias más imperativas de la existencia del campo futbolístico. El cuerpo masculino no se bifurca sino que se afirma aún más dentro de la cancha de fútbol; los valores de la masculinidad se recuerdan cada vez que un hombre pisa el terreno de juego. El sujeto femenino actúa en dos campos al mismo tiempo de igual forma que debe ser eficiente en cada uno de ellos. Se desdobla el cuerpo femenino para replegarse finalmente en la afirmación negociada de su feminidad hegemónica.

\section{Referencias}

Amorós, C. (1994) Espacio público, espacio privado y definiciones ideológicas de lo masculino y lo femenino. Consultado el 13 de febrero de 2008 en www.iidh.ed.cr/ comunidades/derechosmujer/docs/dm_enlinea/espacio\%20publico,\%20espacio\%20privado0173.pdf.
Badinter, E. (1993) XY, La identidad masculina. Bogotá, Norma.

Bourdieu, P. (1988) La distinción. Criterio y bases sociales del gusto. Madrid, Taurus.

$$
\text { drid }
$$

(2000a) Cuestiones de sociología. Istmo, S.A. Ma-

(2000b) La dominación masculina. Anagrama. Colección Argumentos. Barcelona.

Elias, N. Y Dunning, E. (1996) Deporte y ocio en el proceso de civilización. México, FCE.

Lamas, M. (1994) Cuerpo: diferencia sexual y género. Debate feminista. Cuerpo y política, Volumen 10, Año 5. 3-32.

Pedraza, Z. (1999) En cuerpo y alma: Visiones del progreso y la felicidad. Universidad de los Andes, Bogotá.

Rodo, A. (1994) El cuerpo ausente. Debate feminista. Cuerpo y política, Volumen 10, Año 5. 81-95. México.

Ruiz, J. (2003) Representaciones de género en el fútbol femenino. Estudio del fútbol femenino en la Universidad Nacional de Colombia. Bogotá, Universidad Nacional de Colombia, Departamento de Sociología.

Tomlinson, J. (2001) Globalización y cultura. México, Oxford.

Touraine, A. (1992) Critica de la modernidad. México, FCE. 